\title{
Effects of Zinc Status on Age-related T cell Dysfunction and Chronic Inflammation
}

\author{
Carmen P. Wong ${ }^{1,2}$, Kathy R. Magnusson ${ }^{1,3}$, Thomas J. Sharpton ${ }^{4,5}$, Emily Ho ${ }^{*}, 1,2,6$ \\ ${ }^{1}$ Linus Pauling Institute, Oregon State University, OR 97331, USA \\ ${ }^{2}$ School of Biological and Population Health Sciences, Oregon State University, OR 97331, USA \\ ${ }^{3}$ Department of Biomedical Sciences, Carlson College of Veterinary Medicine, Oregon State \\ University, OR 97331, USA \\ ${ }^{4}$ Department of Microbiology, Oregon State University, Corvallis OR 97331, USA \\ ${ }^{5}$ Department of Statistics, Oregon State University, Corvallis OR 97331, USA \\ ${ }^{6}$ Moore Family Center for Whole Grain Foods, Nutrition and Preventive Health, Oregon State \\ University, OR 97331, USA
}

\begin{abstract}
Age-related T cell dysfunction contributes to immunosenescence and chronic inflammation. Aging is also associated with a progressive decline in zinc status. Zinc is an essential micronutrient critical for immune function. A significant portion of the older populations are at risk for marginal zinc deficiency. The combined impact of dietary zinc deficiency and age on immune dysfunction has not been well explored despite the common occurrence together in the elderly population. We hypothesize that age-related zinc loss contributes to $\mathrm{T}$ cell dysfunction and chronic inflammation in the elderly and is exacerabated by inadequate dietary intake and improved with zinc supplementation. Using an aging mouse model, the effects of marginal zinc deficiency and zinc supplementation on, Th1/Th17/proinflammatory cytokine profiles and $\mathrm{CD} 4^{+} \mathrm{T}$ cell naïve/ memory phenotypes were examined. In the first study, young ( $2 \mathrm{mo}$ ) and old (24 mo) C57BL/6 mice were fed a zinc adequate (ZA) or marginally zinc deficient (MZD) diets for 6 wks. In the second study, mice were fed a ZA or zinc supplemented (ZS) diet for $6 \mathrm{wks}$. MZD old mice had a significant increase in LPS-induced IL6 compared to ZA old mice. In contrast, ZS old mice had significantly reduced plasma MCP1 levels, reduced T cell IFN $\gamma$, IL17, and TNFa response, as
\end{abstract}

"Corresponding author: Emily.Ho@oregonstate.edu.

Authors contribution: CPW: Conceptualization, Methodology, Investigation, Validation, Formal analysis, Writing - Original Draft Preparation; KRM: Conceptualization, Writing - Original Draft Preparation; TJS: Conceptualization, Funding acquisition, Methodology, Writing - Original Draft Preparation; EH: Conceptualization, Funding acquisition, Supervision, Methodology, Writing - Original Draft Preparation

Ethics approval: The animal protocol was approved by the Oregon State University Institutional Laboratory Animal Care and Use Committee, and adhered to the international standards of animal care as established by the Association for Assessment and Accreditation of Laboratory Animal Care International.

Availability of data and material: available upon request

Code availability: not applicable

DECLARATIONS

Conflicts of interest/Competing interests: None 
well as increased naïve $\mathrm{CD} 4^{+} \mathrm{T}$-cell subset compared to $\mathrm{ZA}$ old mice. Our data suggest that zinc deficiency is an important contributing factor in immune aging, and improving zinc status can in part reverse immune dysfunction and reduce chronic inflammation associated with aging.

\section{Keywords}

Zinc; aging; inflammation; immune dysfunction; T cells

\section{INTRODUCTION}

Aging is a multi-faceted process that is associated with a myriad of physiological changes over time. One of the hallmarks of aging is the progressive deterioration of the immune system, involving dysfunction in both adaptive and innate immunity which contribute to immunosenescence and chronic inflammation (Fulop et al. 2017; Rea et al. 2018). Among the many age-related immune changes, aging of the $\mathrm{T}$ cell compartment has been studied extensively. Multiple T cell-related defects have been observed with increased age, ranging from altered $\mathrm{T}$ cell development to altered $\mathrm{T}$ cell homeostasis and function in the periphery (Harpaz et al. 2017; Nikolich-Zugich 2014). Age-related T cell dysfunction can contribute to the promotion of chronic inflammatory disease (Goronzy and Weyand 2017). At the same time, a systemic chronic inflammatory environment can also influence the phenotype and function of T cells (Elyahu et al. 2019; Tsukamoto et al. 2015). Recent studies have provided a better understanding of the role of $\mathrm{T}$ cells in the aging process, and how age-related thymic involution and the associated changes in $\mathrm{T}$ cell functionality contribute to immunosenescence and chronic inflammation (Coder et al. 2015; Elyahu et al. 2019; Thomas et al. 2020). Strategies that improve T cell functions and reduce chronic inflammation will be invaluable in promoting healthy aging and reducing age-related morbidities.

Nutritional status plays an important role in promoting healthy aging (High 2001; Pae et al. 2012). Inadequate nutrition in the elderly population is one of many factors contributing to the age-related decline in health, and is associated with increased risks of age-related diseases (Bruins et al. 2019; Shlisky et al. 2017). Nutritional deficiencies, including various vitamins and minerals, are common in older adults. This is due to inadequate intake of nutrient-dense food, as well as age-related inefficiencies in absorption and utilization. Our group has an ongoing interest in understanding the role of zinc in immune health, and how low zinc status may contribute to age-related immune dysfunction, inflammation and associated morbidities. Zinc is a micronutrient that is critical in the development and function of the immune system. It is estimated that $17 \%$ of the world's population is at risk of inadequate zinc intake, with prevalence up to 25-30\% in regions in Sub-Saharan Africa and South Asia (Wessells and Brown 2012). In the US, it is estimated tat $15 \%$ of the US population has a prevalence of inadequacy for zinc. In older individuals this estimate rises to $35-45 \%$, with older adults from households from food-insufficient families have even lower zinc intakes (Dixon et al. 2001; Ervin and Kennedy-Stephenson 2002; Reider et al. 2020). In addition to inadequate dietary zinc intake, the elderly also have reduced zinc absorption that can further lower their zinc status (August et al. 1989; Turnlund et 
al. 1986). The effects of zinc deficiency share many similarities to age-related immune dysfunction, including thymic atrophy, lymphopenia, impaired adaptive immunity, and increased susceptibility to infections (Fischer Walker and Black 2004; Fraker and King 2004). At the same time, low zinc status is associated with dysregulated immune activation, increased oxidative stress, and increased systemic inflammatory response (Gammoh and Rink 2017; Wong et al. 2019; Wong et al. 2015). Reduced zinc status has been observed in the elderly, and the effects of zinc supplementation on immune parameters in elderly subjects have been examined in various studies with inconsistent conclusion (Haase and Rink 2009; Pae and Wu 2017). While the impacts of zinc deficiency on immune function and the correlation between reduced zinc status and aging is well established, the combined impact of dietary zinc deficiency and age on immune dysfunction has not been well defined despite the common occurrence together in this at risk population. In particular, whether dietary zinc deficiency exacerbates age-related immune dysfunction is unclear. The aging mouse model is a valuable resource in understanding aging and age-related diseases, and shares many of the immune aging hallmarks observed in humans, including decreased naïve T-cell populations as well as increased proinflammatory cytokine response (Maue et al. 2009; Mestas and Hughes 2004). This model allows us to address pending/unanswered questions regarding the respective impacts of age-related zinc deficiency (e.g. impaired zinc absorption with age) versus dietary zinc deficiency (due to inadequate zinc intake), as well as their combined impact on immune dysfunction. Our group had previously shown in aged mice that various immune populations had reduced intracellular zinc with age (Wong et al. 2013). We further showed that zinc supplementation can increase zinc status and improve thymopoiesis in aged mice (Wong et al. 2009). Whether dietary zinc deficiency exacerbates age-related immune dysfunction is not well established. At the same time, it remains unclear whether improved thymopoiesis with zinc supplementation will translate to improved T cell function in the periphery, and/or reverse some of the $\mathrm{T}$ cell defects observed with aging. In this study, we examined the interaction between dietary zinc intake and age-related decline in zinc status, and their consequence on immune aging parameters. The goal of this study was to determine the effects of marginal zinc deficiency and zinc supplementation on changes in $\mathrm{T}$ cell phenotype, cytokine profiles, and inflammatory response in young and old mice. We hypothesized that low zinc status is an important contributing factor in age-related immune dysfunction and chronic inflammation, and improving zinc status via zinc supplementation will alleviate some of the age-related immune dysfunction.

\section{MATERIAL AND METHODS}

\subsection{Animal Study Design}

Old (23 mo) C57BL/6 male mice were obtained from the National Institute of Aging (NIA) aged rodent colony. Young (1 mo) C57BL/6 male mice were purchased from Jackson Laboratories (Bar Harbor, ME), which is the source for the NIA colony. Due to restrictions in the availability of old mice from NIA (10 old mice available every two months), we have designed our study to examine the effects of marginal zinc deficiency and the effects of zinc supplementation, using separate cohorts of mice, each with its own control (zinc adequate) group (see details described below). Only male mice were used in this study due to limitations as stated above. Upon arrival, all mice were acclimated to the control 
zinc adequate diet (see diet details below) for 1 mo prior to the beginning of the study. After acclimation, young $(2 \mathrm{mo})$ and old mice $(24 \mathrm{mo})$ were randomly assigned to different dietary treatments for $6 \mathrm{wks}$ (10 mice per age group for each dietary treatment). All mice were individually housed in ventilated microisolater cages and kept in a temperature- and humidity-controlled environment $\left(22^{\circ} \mathrm{C}, 50 \%\right.$ humidity, 12 -h light cycle). None of the old mice had any obvious signs of illness, tumors or lesions at the beginning of the study. To study the effects of marginal zinc deficiency young and old mice were fed either a purified diet containing $30 \mathrm{mg} / \mathrm{kg}$ zinc (zinc adequate, ZA) or $6 \mathrm{mg} / \mathrm{kg}$ zinc (marginally zinc deficient, MZD) for 6 wks. To study the effects of zinc supplementation, a separate cohort of young and old mice were fed either a purified diet containing $30 \mathrm{mg} / \mathrm{kg}$ zinc (ZA) or $300 \mathrm{mg} / \mathrm{kg}$ zinc (zinc supplemented, ZS) for 6 wks. Each age by diet group contained 10 individuals. Diets were custom formulated using a modified egg white-based AIN-93G diet with zinc provided as zinc carbonate. Purified ZA, MZD, and ZS diets were purchased from Research Diets (New Brunswick, NJ). MZD diet and ZS diet had previously been shown to result in reduced and increased zinc status in mice, respectively (Wong et al. 2019; Wong et al. 2009). Food and water were provided ad libitum, and food intakes and body weights of all mice were monitored throughout the study. There were no differences in food intake and body weight due to the different dietary treatments (data not shown). Estimated daily doses of zinc consumed per animal (in $\mathrm{mg}$ zinc/kg body weight \pm SEM) were as follows: young mice fed ZA diet $(2.87 \pm 0.05)$, old mice fed ZA diet $(2.81 \pm 0.17)$, young mice fed MZD $\operatorname{diet}(0.53 \pm 0.02)$, old mice fed MZD diet $(0.49 \pm 0.01)$, young mice fed ZS diet $(30.10$ \pm 0.93 ), old mice fed ZS diet (26.64 \pm 0.60 ). At the beginning (wk 0) and end (wk 6) of study, whole blood samples were collected from the submandibular vein in all mice, with the use of Goldenrod animal lancets (Braintree Scientific, Braintree, MA), into BD Microtainer heparin blood collection tubes (BD Biosciences, Franklin Lakes, NJ). Week 6 whole blood samples were collected 2-3 days prior to euthanasia at the end of study. At the termination of the experiments, mice were euthanized by $\mathrm{CO}_{2}$ asphyxiation, and sera and tissues were collected. The animal protocol was approved by the Oregon State University Institutional Laboratory Animal Care and Use Committee (Animal Care and Use Protocol \#4996), and adhered to the international standards of animal care as established by the Association for Assessment and Accreditation of Laboratory Animal Care International.

\subsection{Serum zinc measurements}

Serum zinc concentrations were determined by inductively coupled plasma-optical emission spectroscopy (ICP-OES), as previously described (Wong et al. 2009). Samples were digested overnight in $0.5 \mathrm{ml}$ nitric acid (OmniTrace® nitric acid, EMD Millipore, Billerica, MA), and diluted with Chelex-treated nanopure water to a final concentration of $10 \%(\mathrm{v} / \mathrm{v})$ nitric acid followed by centrifugation to remove particulates. Samples were analyzed using the Prodigy High Dispersion ICP-OES instrument (Teledyne Leeman Labs, Hudson, NH) against known standards. ICP-OES analyses were done at the W.M. Keck Collaboratory for Plasma Spectrometry at Oregon State University.

\subsection{Whole blood inflammatory cytokines production}

Biomarkers for chronic inflammation in the aging mouse model included, among others, elevated serum MCP1 and LPS-induced IL6 in whole blood (Conley et al. 2016; 
Thevaranjan et al. 2017). Quantification of various inflammatory cytokines in whole blood was done as previously described with slight modifications (Rodriguez-Caballero et al. 2004; Schuerwegh et al. 2003). Heparinized whole blood samples (50 $\mu \mathrm{l})$ were diluted 1:1 with $50 \mu$ complete RPMI media (RPMI media supplemented with $10 \%$ FBS, penicillinstreptomycin, and $50 \mu \mathrm{M} \beta$-mercaptoethanol). Diluted blood samples were left unstimulated, or stimulated with $10 \mathrm{ng} / \mathrm{ml}$ LPS in 96-well half area tissue culture plates in humidified incubators with $5 \% \mathrm{CO} 2$ at $37{ }^{\circ} \mathrm{C}$. Cell-free culture supernatants were collected after $24 \mathrm{~h}$. Production of inflammatory cytokines (IL6, IL10, IL12, IFN $\gamma$, MCP1, and TNFa) were determined using BD Cytometric Bead Array (CBA) Mouse Inflammation Kit (BD Biosciences).

\subsection{Ex vivo T cell activation and Th1/Th2/Th17 cytokine production}

Single cell suspensions were prepared from mesenteric and inguinal lymph nodes (LN) collected from individual mice, and adjusted to $2 \times 10^{6}$ cells $/ \mathrm{ml}$ in complete RPMI media. Equal number of LN cells $\left(2 \times 10^{5}\right.$ cells) were seeded in 96-well round bottom tissue culture plates, and stimulated with either plate-bound anti-CD3 $(5 \mu \mathrm{g} / \mathrm{ml})$ and soluble anti-CD28 (2 $\mu \mathrm{g} / \mathrm{ml})$, or PMA ( $25 \mathrm{ng} / \mathrm{ml})$ and ionomycin $(1 \mu \mathrm{g} / \mathrm{ml})$ in humidified incubators with $5 \% \mathrm{CO} 2$ at $37{ }^{\circ} \mathrm{C}$. Unstimulated cells were used as negative controls. Cell-free culture supernatants were collected after $24 \mathrm{~h}$ (PMA/ionomycin) or 48h (anti-CD3/anti-CD28). Production of activation-induced Th1/Th2/Th17 cytokines (IL2, IL4, IL6, IL10, IL17, IFN $\gamma$, and TNFa) were determined using BD CBA Mouse Th1/Th2/Th17 Kit (BD Biosciences).

\subsection{Flow cytometry analyses}

For flow cytometry analysis, LN from individual mice were made into a single cell suspension in flow cytometry buffer (PBS, 2\% FBS, 1 mM EDTA). CD4 and CD8 T cell subsets, as well as naïve and memory $\mathrm{T}$ cell populations were identified using antibodies specific against CD4, CD8, CD44, and CD62L. Regulatory T cells (Treg) were identified based on CD4 and FoxP3 expression using mouse regulatory T cells staining kit. All antibodies and staining kits were purchased from eBioscience (San Diego, CA). Labeled cells were washed twice and resuspended in buffer for flow cytometry acquisition and analysis. A minimum of 10,000 cells in the lymphocyte gate (based on forward and side scatter) were collected. Data were acquired using FACSCalibur (BD Biosciences) and analyzed using Summit software (DakoCytomation, Fort Collins, CO).

\subsection{Statistical analyses}

Statistical analyses were done using GraphPad Prism Version 7.04 (Graph-Pad, La Jolla, CA). Two-way ANOVA was used to determine the significance of main effects of zinc status and age and their interaction, followed by Tukey's multiple comparisons test where appropriate. Normality of data was determined using the Shapiro-Wilk normality test. Where necessary, data were log transformed to correct for unequal variances prior to statistical analyses. Non-transformed data are shown in all figures. Statistical significance is defined as $P \leq 0.05$. 


\section{RESULTS}

\subsection{Marginal zinc deficiency reduced zinc status and further increased LPS-induced IL6 response in old mice}

The effect of MZD on zinc status and inflammation was determined in young ( $2 \mathrm{mo})$ and old (24 mo) mice fed a ZA or MZD diet. Despite being fed a ZA diet, old mice had significant reduced serum zinc levels compared to young mice, and the serum zinc levels were further decreased in old mice fed MZD diet (Fig. 1A). Increased systemic inflammation, including elevated LPS-induced IL6, is associated with age (Thevaranjan et al. 2017). The effect of reduced zinc status on LPS-induced IL6 was determined in whole blood samples from individual mice at the beginning ( $\mathrm{wk} 0$ ) and end of the study (wk 6). Significant age-related increase in LPS-induced IL6 was observed in old mice at wk 0 (Fig. 1B). MZD exposures for 6wks significantly increased LPS-induced IL6 response, as well as exhibit a significant interaction effect between age and zinc status in further enhancing LPSinduced IL6 response in the older mice (Fig. 1C). While systemic inflammatory response was increased with lower zinc status, age-related increase in Th1/Th17 T cell cytokine response were not exacerbated in MZD old mice (data not shown).

\subsection{Zinc supplementation increased zinc status and reduced MCP1 in old mice}

We next determined the effect of ZS on zinc status and inflammation in young and old mice fed ZA or ZS diet. Old mice fed ZS diet had improved serum zinc levels that were comparable to those observed in young mice fed a ZA diet (Fig. 2A). MCP1, a proinflammatory cytokine that is elevated with age (Conley et al. 2016), was measured in whole blood samples from individual mice at the beginning of the study (wk 0) and at the end of the study (wk 6). Significant age-related increase in MCP1 was observed in old mice at the beginning of the study (Fig. 2B). After 6 wks dietary treatment, ZS had a significant effect on reducing MCP1 levels, with the data collapsed across ages (Fig. 2C). Increased zinc status did not alter LPS-induced IL6 response (data not shown).

\subsection{Zinc supplementation increased naïve $\mathrm{CD}^{+} \mathrm{T}$ cells subset and decreased activation- induced Th1/Th17/proinflammatory cytokines production in old mice}

In mice, it is well established that $\mathrm{T}$ cell function and phenotype are altered with age, as demonstrated by increased Th1/Th17 responses, reduced naïve T cells with concomitant increased memory T cells, and skewed CD4/CD8 T cell ratio, among others (Elyahu et al. 2019; Harpaz et al. 2017; Pinchuk and Filipov 2008; Swain et al. 2005). The effects of zinc supplementation on $\mathrm{CD} 4 / \mathrm{CD} 8$ ratio, naïve $\mathrm{CD}^{+} \mathrm{T}$ cell populations, Treg, and activationinduced Th1/Th17/proinflammatory cytokine response were determined in lymph nodes (LNs) of young and old mice. As expected, skewed CD4/CD8 ratio and decreased CD4 ${ }^{+}$ naïve $T$ cell subset were observed in old mice fed ZA diet (Fig. 3). Improving zinc status did not reverse age-related skewing of CD4/CD8 T cell ratio in old mice (Fig. 3A). However, it had a significant effect in increasing naïve $\mathrm{CD} 4^{+} \mathrm{T}$ cell populations in old mice fed $\mathrm{ZS}$ diet (Fig. 3B). Age-related increase in Treg was not reduced with zinc supplementation. The percentage of LN Tregs, as determined by CD4 and FoxP3 expression, were as follows: ZA young mice $(14.81 \pm 0.5)$, ZA old mice $(26.1 \pm 3.6)$, ZS young mice $(14.0 \pm 0.6)$, and ZS old mice $(25.3 \pm 3.0)$. Upon $\mathrm{T}$ cell activation with either anti-CD3/CD28 or PMA/ionomycin, 
lymphocytes from ZA old mice had significant age-related increases in activation-induced Th1 (IFN $\gamma$ ), Th17 (IL17), and proinflammatory (TNFa) cytokines compared to young mice (Fig. 4). Increasing zinc status in ZS old mice significantly decreased activation-induced IFN $\gamma$, IL17, and TNFa production compared to cells from old mice fed ZA diet (Fig. 4). A significant diet effect as well as significant interactions between age and diet were observed in anti-CD3/CD28 activated lymphocytes, and/or PMA/ionomycin activated lymphocytes.

\section{DISCUSSION}

Aging of the immune system is a complex biological process, involving numerous changes in both adaptive and innate immune system. Both immunosenescence and chronic inflammation contribute to the increased risk for age-related diseases. While it is clear that immune dysfunction occurs with age, there remains a critical need to understand factors that impact susceptibility to immunosesenecence to help develop strategies to improve/rejuvenate immune functions in the aging population. Intervention strategies to reverse the immune decline and dysfunction require an understanding of modifiable factors that can be restored in the immune aging process. Nutrition status is one such factor that can affect immunity, and nutritional insufficiency and deficiency commonly observed in the elderly populations can contribute and/or exacerbate immune impairments observed with age (Wu et al. 2018). Reversal or correction of nutrient deficiencies is one potential approach to restore immune function in the elderly (Aspinall and Lang 2018). Findings from our current study suggest that zinc status is an important nutritional factor that can modulate age-related immune response. Our work suggests that improving zinc status via dietary intervention in the aged mice may, in part, reverse some of the immune impairments in the T cell compartment as well as lessen chronic inflammation associated with age. Optimizing and ensuring adequate zinc intake in the older populations can be a potential intervention strategy in improving the aged immune system.

The aging mouse model is a valuable resource in understanding aging and age-related diseases, and allows us to directly examine changes in lymphoid tissues that are neither accessible nor practical to examine in human subjects. Differences exist in both innate and adaptive immunity between mice and human, and need to be taken into careful consideration when using mice as an aging model (Maue et al. 2009; Mestas and Hughes 2004). Nevertheless immune aging in a rodent model shares many similarities with humans, including thymic involution, impaired adaptive immune response, altered $\mathrm{T}$ cell cytokine profiles, and elevated inflammatory markers, among others, and allow us to bypass inherent limitations in human subjects. Importantly, the decline in zinc status observed in elderly human population is similarly observed in aged mice (Haase et al. 2006; Wong et al. 2013). The primary objective of this study was to examine the potential interaction between dietary zinc status and aging on chronic inflammation and $\mathrm{T}$ cell subset/cytokine dysfunction. We and others had previously shown that restoring zinc status via dietary zinc supplementation partially reversed thymic involution and reduced age-associated inflammation in old mice (Dardenne et al. 1993; Wong et al. 2013; Wong et al. 2009). However, whether improved thymic activities with zinc supplementation would translate to improved peripheral $\mathrm{T}$ cells development and function was unknown. In young mice and rats, zinc deficiency results in increased oxidative stress, inflammatory response, and DNA damage (Song et al. 2009; 
Wong et al. 2019). It was unclear if a further decrease in zinc status in old mice would worsen age-related immune dysfunction. In our study, old mice fed a MZD diet did not show further increases in activation-induced IFN $\gamma$, IL17, and TNFa cytokines production in LN $\mathrm{T}$ cells (data not shown). While intrinsic $\mathrm{T}$ cell defects with age were not exacerbated with further reduction in zinc status, LPS-induced IL6 response in old mice was further increased by marginal zinc deficiency (Fig. 1). Findings from a recent study suggested the LPS could induce the formation of senescent macrophages, characterized by the morphological changes and senescence-associated secretory phenotype, and persistent DNA damage response (Wang et al. 2020). It would be of interest to determine if zinc deficiency results in the accumulation of senescent cells in old mice. Recent work suggested that zinc deficiency could increase sensitivity to arsenic toxicity, resulting in elevated proinflammatory response and oxidative stress (Wong et al. 2019). Our data suggested that zinc deficiency could similarly increase vulnerability to endotoxin stress, which is elevated with age (Kohman et al. 2010; Tateda et al. 1996). When zinc status in old mice was restored to levels comparable to those in young mice via zinc supplementation, various immune parameters were improved, including decreased systemic MCP1 (Fig. 2), increased naïve CD4 ${ }^{+} \mathrm{T}$ cells (Fig. 3), and decreased activation-induced IFN $\gamma$, IL17, and TNFa cytokine production in LN T cells (Fig. 4). While these improvements did not approach levels observed in young mice, our data provided evidence that increasing zinc status via dietary intervention could reverse some of the immune dysfunction observed with aging, particularly in the $\mathrm{T}$ cell compartment. It should be noted, however, that not all inflammatory biomarkers that were elevated with age could be corrected with zinc supplementation (e.g. LPS-induced IL-6), and it would be of interest to further investigate the differential effects of zinc on different inflammatory pathways.

In human clinical studies, the effects of zinc supplementation on improving immune functions during the course of viral and bacterial infections, as well as in the elderly populations have yielded inconsistent results. While some studies showed improved immune functions and reduced incidence of infections (Fortes et al. 1998; Girodon et al. 1999; Girodon et al. 1997; Meydani et al. 2007), others showed no effect on immune parameters (Bogden et al. 1988; Hodkinson et al. 2007; Provinciali et al. 1998). The lack of consistency in study outcomes were likely attributed to the varying health and zinc status of individuals, as well as differences in dosage, study durations, age of study populations, and immune parameters measured, details of various studies summarized in previous reviews (Haase and Rink 2009; Pae and Wu 2017). Genotype differences in individuals e.g. polymorphisms in genes encoding zinc transporters can also potentially influence zinc uptake and efficacy of zinc supplementation (Day et al. 2017). In addition, the lack of sensitive and specific zinc biomarkers to determine zinc status in individuals also hampers the interpretation of clinical results (King et al. 2015). The development of better zinc biomarkers will allow for more accurate assessment of zinc status and evaluation of the efficacy of zinc supplementation in at risk individuals. In our animal studies, future work will need to be done to determine if a longer duration and/or increasing dosage of zinc supplementation will have additional benefits in improving/restoring immune functions in the old mice. The potential benefits of a longer term study will need to be balanced with the increasing variability in health with increasing age in mice. There still exist gaps in knowledge in optimal zinc doses and timing, 
and sources of variability in individual responses with advanced age. Moreover, additional studies to determine the benefits of earlier intervention (e.g. zinc supplementation in middle aged mice), and a more in depth analysis of the effects of zinc supplementation on the function and phenotype of individual $\mathrm{T}$ cell subsets, would allow us to better understand and clarify zinc's mechanisms of action. In addition, a better understanding of the interaction between zinc and other factors that contribute to immunity, would allow us to better define and clarify zinc's mechanisms of action. For example, increasing evidence suggest the importance of gut microbiome on immune development and chronic inflammation in the context of aging, where age-related gut dysbiosis may contribute to unhealthy aging (Kim and Jazwinski 2018; Tibbs et al. 2019). Understanding the potential interaction between zinc status and gut microbiome would be an important future area of study.

The importance of the micronutrient zinc on immune development and function, as well as the negative impact of zinc deficiency on immunity, are well established. Increasing evidence suggested that the progressive decline in zinc status with age may contribute to age-related immune dysfunction and chronic inflammation, and improving zinc status in the elderly will improve immune function and reduce chronic inflammation. Our data suggested that zinc status was an important contributing factor in age-related immune dysfunction and chronic inflammation, and zinc supplementation could in part restore/improve the function and composition of $\mathrm{T}$ cells in aged mice. Modulation of nutrition status is a potential intervention strategy in improving and restoring immune function in the elderly.

\section{ACKNOWLEDGEMENTS}

We would like to thank Dr. Adam Branscum, Professor and Program Director of Biostatistics at Oregon State University for providing expert consultation regarding statistical analysis approach and data interpretation. This work was supported by United States Department of Agriculture National Institute of Food and Agriculture (NIFA-2018-67017-27358), as well as funding from Oregon Agricultural Experimental Station (OR00735).

Funding: United States Department of Agriculture National Institute of Food and Agriculture (NIFA-2018-67017-27358), and Oregon Agricultural Experimental Station (OR00735)

\section{Abbreviations:}

CBA

ICP-OES

LN

MZD

NIA

Treg

ZA

ZS
Cytometric Bead Array

inductively coupled plasma-optical emission spectroscopy

lymph nodes

marginally zinc deficient

National Institute of Aging

regulatory $\mathrm{T}$ cells

zinc adequate

zinc supplemented 


\section{REFERENCES}

Aspinall R, Lang PO (2018) Interventions to restore appropriate immune function in the elderly Immun Ageing 15:5 doi:10.1186/s12979-017-0111-6 [PubMed: 29416551]

August D, Janghorbani M, Young VR (1989) Determination of zinc and copper absorption at three dietary $\mathrm{Zn}-\mathrm{Cu}$ ratios by using stable isotope methods in young adult and elderly subjects Am J Clin Nutr 50:1457-1463 doi:10.1093/ajcn/50.6.1457 [PubMed: 2596436]

Bogden JD et al. (1988) Zinc and immunocompetence in elderly people: effects of zinc supplementation for 3 months Am J Clin Nutr 48:655-663 doi:10.1093/ajcn/48.3.655 [PubMed: 3414581]

Bruins MJ, Van Dael P, Eggersdorfer M (2019) The Role of Nutrients in Reducing the Risk for Noncommunicable Diseases during Aging Nutrients 11:85 doi:10.3390/nu11010085

Coder BD, Wang H, Ruan L, Su DM (2015) Thymic involution perturbs negative selection leading to autoreactive T cells that induce chronic inflammation J Immunol 194:5825-5837 doi:10.4049/ jimmunol.1500082 [PubMed: 25957168]

Conley MN, Wong CP, Duyck KM, Hord N, Ho E, Sharpton TJ (2016) Aging and serum MCP-1 are associated with gut microbiome composition in a murine model PeerJ 4:e1854 doi:10.7717/ peerj.1854 [PubMed: 27069796]

Dardenne M, Boukaiba N, Gagnerault MC, Homo-Delarche F, Chappuis P, Lemonnier D, Savino W (1993) Restoration of the thymus in aging mice by in vivo zinc supplementation Clin Immunol Immunopathol 66:127-135 doi:10.1006/clin.1993.1016 [PubMed: 8453784]

Day KJ, Adamski MM, Dordevic AL, Murgia C (2017) Genetic Variations as Modifying Factors to Dietary Zinc Requirements-A Systematic Review Nutrients 9 doi:10.3390/nu9020148

Dixon LB, Winkleby MA, Radimer KL (2001) Dietary intakes and serum nutrients differ between adults from food-insufficient and food-sufficient families: Third National Health and Nutrition Examination Survey, 1988-1994 J Nutr 131:1232-1246 doi:10.1093/jn/131.4.1232 [PubMed: 11285332]

Elyahu Y et al. (2019) Aging promotes reorganization of the CD4 T cell landscape toward extreme regulatory and effector phenotypes Sci Adv 5:eaaw8330 doi:10.1126/sciadv.aaw8330

Ervin RB, Kennedy-Stephenson J (2002) Mineral intakes of elderly adult supplement and nonsupplement users in the third national health and nutrition examination survey J Nutr 132:34223427 doi:10.1093/jn/132.11.3422 [PubMed: 12421862]

Fischer Walker C, Black RE (2004) Zinc and the risk for infectious disease Annu Rev Nutr 24:255275 doi:10.1146/annurev.nutr.23.011702.073054 [PubMed: 15189121]

Fortes C et al. (1998) The effect of zinc and vitamin A supplementation on immune response in an older population J Am Geriatr Soc 46:19-26 doi:10.1111/j.1532-5415.1998.tb01008.x [PubMed: 9434661]

Fraker PJ, King LE (2004) Reprogramming of the immune system during zinc deficiency Annu Rev Nutr 24:277-298 doi:10.1146/annurev.nutr.24.012003.132454 [PubMed: 15189122]

Fulop T, Larbi A, Dupuis G, Le Page A, Frost EH, Cohen AA, Witkowski JM, Franceschi C (2017) Immunosenescence and Inflamm-Aging As Two Sides of the Same Coin: Friends or Foes? Front Immunol 8:1960 doi:10.3389/fimmu.2017.01960 [PubMed: 29375577]

Gammoh NZ, Rink L (2017) Zinc in Infection and Inflammation Nutrients 9:624 doi:10.3390/ nu9060624

Girodon F, Galan P, Monget AL, Boutron-Ruault MC, Brunet-Lecomte P, Preziosi P, Arnaud J, Manuguerra JC, Herchberg S (1999) Impact of trace elements and vitamin supplementation on immunity and infections in institutionalized elderly patients: a randomized controlled trial. MIN. VIT. AOX. geriatric network Arch Intern Med 159:748-754 doi:10.1001/archinte.159.7.748 [PubMed: 10218756]

Girodon F, Lombard M, Galan P, Brunet-Lecomte P, Monget AL, Arnaud J, Preziosi P, Hercberg S (1997) Effect of micronutrient supplementation on infection in institutionalized elderly subjects: a controlled trial Ann Nutr Metab 41:98-107 doi:10.1159/000177984 [PubMed: 9267584]

Goronzy JJ, Weyand CM (2017) Successful and Maladaptive T Cell Aging Immunity 46:364-378 doi:10.1016/j.immuni.2017.03.010 [PubMed: 28329703] 
Haase H, Mocchegiani E, Rink L (2006) Correlation between zinc status and immune function in the elderly Biogerontology 7:421-428 doi:10.1007/s10522-006-9057-3 [PubMed: 16953331]

Haase H, Rink L (2009) The immune system and the impact of zinc during aging Immun Ageing 6:9 doi:10.1186/1742-4933-6-9 [PubMed: 19523191]

Harpaz I, Bhattacharya U, Elyahu Y, Strominger I, Monsonego A (2017) Old Mice Accumulate Activated Effector CD4 T Cells Refractory to Regulatory T Cell-Induced Immunosuppression Front Immunol 8:283 doi:10.3389/fimmu.2017.00283 [PubMed: 28382033]

High KP (2001) Nutritional strategies to boost immunity and prevent infection in elderly individuals Clin Infect Dis 33:1892-1900 doi:10.1086/324509 [PubMed: 11692301]

Hodkinson CF et al. (2007) Effect of zinc supplementation on the immune status of healthy older individuals aged 55-70 years: the ZENITH Study J Gerontol A Biol Sci Med Sci 62:598-608 doi:10.1093/gerona/62.6.598 [PubMed: 17595415]

Kim S, Jazwinski SM (2018) The Gut Microbiota and Healthy Aging: A Mini-Review Gerontology 64:513-520 doi:10.1159/000490615 [PubMed: 30025401]

King JC, Brown KH, Gibson RS, Krebs NF, Lowe NM, Siekmann JH, Raiten DJ (2015) Biomarkers of Nutrition for Development (BOND)-Zinc Review J Nutr 146:858S-885S doi:10.3945/ jn.115.220079 [PubMed: 26962190]

Kohman RA, Crowell B, Kusnecov AW (2010) Differential sensitivity to endotoxin exposure in young and middle-age mice Brain Behav Immun 24:486-492 doi:10.1016/j.bbi.2009.12.004

Maue AC, Yager EJ, Swain SL, Woodland DL, Blackman MA, Haynes L (2009) T-cell immunosenescence: lessons learned from mouse models of aging Trends Immunol 30:301-305 doi:10.1016/j.it.2009.04.007

Mestas J, Hughes CC (2004) Of mice and not men: differences between mouse and human immunology J Immunol 172:2731-2738 doi:10.4049/jimmunol.172.5.2731

Meydani SN, Barnett JB, Dallal GE, Fine BC, Jacques PF, Leka LS, Hamer DH (2007) Serum zinc and pneumonia in nursing home elderly Am J Clin Nutr 86:1167-1173 doi:10.1093/ajcn/86.4.1167 [PubMed: 17921398]

Nikolich-Zugich J (2014) Aging of the T cell compartment in mice and humans: from no naive expectations to foggy memories J Immunol 193:2622-2629 doi:10.4049/jimmunol.1401174 [PubMed: 25193936]

Pae M, Meydani SN, Wu D (2012) The role of nutrition in enhancing immunity in aging Aging Dis 3:91-129 [PubMed: 22500273]

Pae M, Wu D (2017) Nutritional modulation of age-related changes in the immune system and risk of infection Nutr Res 41:14-35 doi:10.1016/j.nutres.2017.02.001 [PubMed: 28577789]

Pinchuk LM, Filipov NM (2008) Differential effects of age on circulating and splenic leukocyte populations in C57BL/6 and BALB/c male mice Immun Ageing 5:1 doi:10.1186/1742-4933-5-1 [PubMed: 18267021]

Provinciali M et al. (1998) Effect of zinc or zinc plus arginine supplementation on antibody titre and lymphocyte subsets after influenza vaccination in elderly subjects: a randomized controlled trial Age Ageing 27:715-722 doi:10.1093/ageing/27.6.715

Rea IM, Gibson DS, McGilligan V, McNerlan SE, Alexander HD, Ross OA (2018) Age and Age-Related Diseases: Role of Inflammation Triggers and Cytokines Front Immunol 9:586 doi:10.3389/fimmu.2018.00586 [PubMed: 29686666]

Reider CA, Chung RY, Devarshi PP, Grant RW, Hazels Mitmesser S (2020) Inadequacy of Immune Health Nutrients: Intakes in US Adults, the 2005-2016 NHANES Nutrients 12 doi:10.3390/ nu12061735

Rodriguez-Caballero A, Garcia-Montero AC, Bueno C, Almeida J, Varro R, Chen R, Pandiella A, Orfao A (2004) A new simple whole blood flow cytometry-based method for simultaneous identification of activated cells and quantitative evaluation of cytokines released during activation Lab Invest 84:1387-1398 doi:10.1038/labinvest.3700162 [PubMed: 15311213]

Schuerwegh AJ, De Clerck LS, Bridts CH, Stevens WJ (2003) Comparison of intracellular cytokine production with extracellular cytokine levels using two flow cytometric techniques Cytometry B Clin Cytom 55:52-58 doi:10.1002/cyto.b.10041 
Shlisky J et al. (2017) Nutritional Considerations for Healthy Aging and Reduction in Age-Related Chronic Disease Adv Nutr 8:17-26 doi:10.3945/an.116.013474 [PubMed: 28096124]

Song Y, Leonard SW, Traber MG, Ho E (2009) Zinc deficiency affects DNA damage, oxidative stress, antioxidant defenses, and DNA repair in rats J Nutr 139:1626-1631 doi:10.3945/jn.109.106369 [PubMed: 19625698]

Swain S, Clise-Dwyer K, Haynes L (2005) Homeostasis and the age-associated defect of CD4 T cells Semin Immunol 17:370-377 doi:10.1016/j.smim.2005.05.007 [PubMed: 15964201]

Tateda K, Matsumoto T, Miyazaki S, Yamaguchi K (1996) Lipopolysaccharide-induced lethality and cytokine production in aged mice Infect Immun 64:769-774 [PubMed: 8641780]

Thevaranjan N et al. (2017) Age-Associated Microbial Dysbiosis Promotes Intestinal Permeability, Systemic Inflammation, and Macrophage Dysfunction Cell Host Microbe 21:455-466 e454 doi:10.1016/j.chom.2017.03.002 [PubMed: 28407483]

Thomas R, Wang W, Su DM (2020) Contributions of Age-Related Thymic Involution to Immunosenescence and Inflammaging Immun Ageing 17:2 doi:10.1186/s12979-020-0173-8 [PubMed: 31988649]

Tibbs TN, Lopez LR, Arthur JC (2019) The influence of the microbiota on immune development, chronic inflammation, and cancer in the context of aging Microb Cell 6:324-334 doi:10.15698/ mic2019.08.685 [PubMed: 31403049]

Tsukamoto H, Senju S, Matsumura K, Swain SL, Nishimura Y (2015) IL-6-mediated environmental conditioning of defective Th1 differentiation dampens antitumour immune responses in old age Nat Commun 6:6702 doi:10.1038/ncomms7702 [PubMed: 25850032]

Turnlund JR, Durkin N, Costa F, Margen S (1986) Stable isotope studies of zinc absorption and retention in young and elderly men J Nutr 116:1239-1247 doi:10.1093/jn/116.7.1239 [PubMed: 3746461]

Wang $\mathrm{H}$ et al. (2020) BRD4 contributes to LPS-induced macrophage senescence and promotes progression of atherosclerosis-associated lipid uptake Aging (Albany NY) 12:9240-9259 doi:10.18632/aging.103200 [PubMed: 32392533]

Wessells KR, Brown KH (2012) Estimating the global prevalence of zinc deficiency: results based on zinc availability in national food supplies and the prevalence of stunting PLoS One 7:e50568 doi:10.1371/journal.pone.0050568 [PubMed: 23209782]

Wong CP, Dashner-Titus EJ, Alvarez SC, Chase TT, Hudson LG, Ho E (2019) Zinc Deficiency and Arsenic Exposure Can Act Both Independently or Cooperatively to Affect Zinc Status, Oxidative Stress, and Inflammatory Response Biol Trace Elem Res 191:370-381 doi:10.1007/ s12011-019-1631-z [PubMed: 30635848]

Wong CP, Magnusson KR, Ho E (2013) Increased inflammatory response in aged mice is associated with age-related zinc deficiency and zinc transporter dysregulation J Nutr Biochem 24:353-359 doi:10.1016/j.jnutbio.2012.07.005 [PubMed: 22981370]

Wong CP, Rinaldi NA, Ho E (2015) Zinc deficiency enhanced inflammatory response by increasing immune cell activation and inducing IL6 promoter demethylation Mol Nutr Food Res 59:991-999 doi:10.1002/mnfr.201400761 [PubMed: 25656040]

Wong CP, Song Y, Elias VD, Magnusson KR, Ho E (2009) Zinc supplementation increases zinc status and thymopoiesis in aged mice J Nutr 139:1393-1397 doi:10.3945/jn.109.106021 [PubMed: 19474155]

Wu D, Lewis ED, Pae M, Meydani SN (2018) Nutritional Modulation of Immune Function: Analysis of Evidence, Mechanisms, and Clinical Relevance Front Immunol 9:3160 doi:10.3389/ fimmu.2018.03160 [PubMed: 30697214] 
A

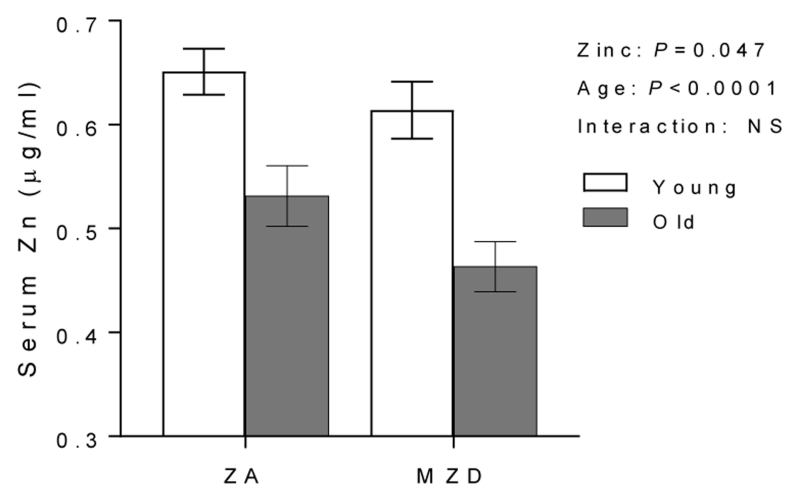

B
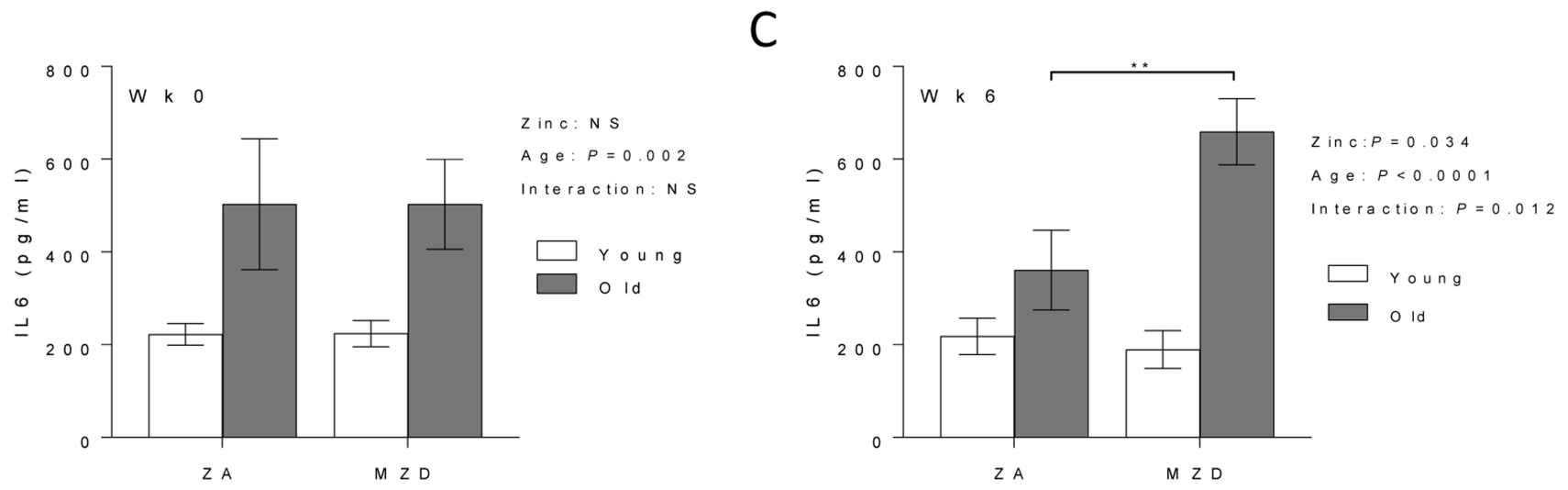

Figure 1. Marginal zinc deficiency deceased zinc status and further increased whole blood LPS-induced IL6 response in old mice.

Young (2 mo) and old (24 mo) C57BL/6 mice were fed a zinc adequate (ZA) diet or marginally zinc deficient (MZD) diet for $6 \mathrm{wks}$. Serum zinc levels were measured by ICP-OES at the end of the study (A). Whole blood were collected at the beginning (wk 0) (B) and end of study (wk 6) (C) to determine LPS-induced IL6 response after 24h ex vivo LPS stimulation $(10 \mathrm{ng} / \mathrm{ml})$. Data represent mean \pm SEM $(n=7-10$ per treatment group). Two-way ANOVA was used to test for main effects of zinc status and age and their interaction, followed by Tukey's multiple comparisons test where appropriate. $* * P<0.01$, NS = not significant. 


\section{A}

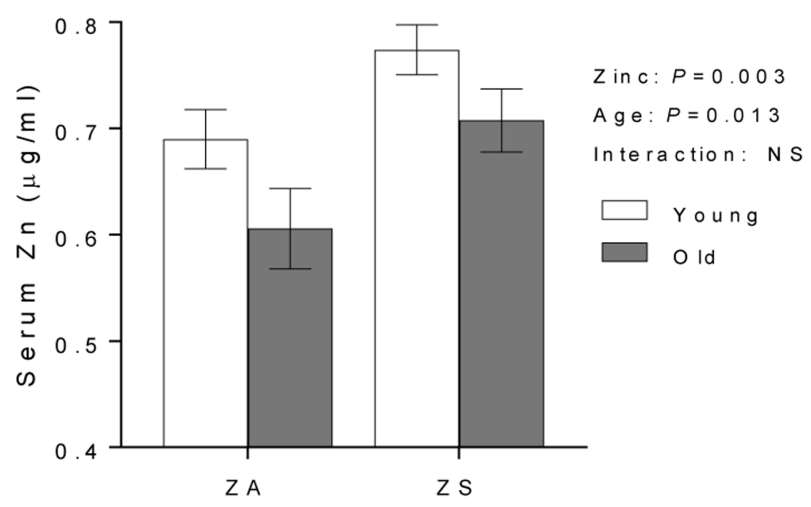

B

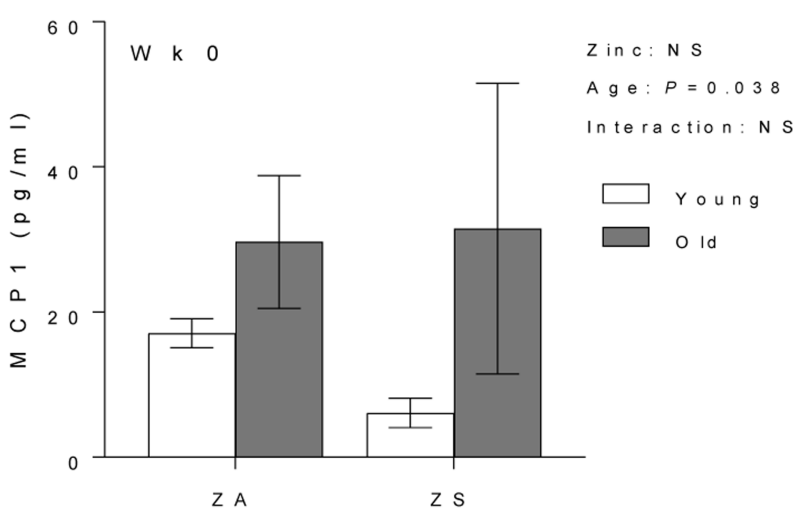

C

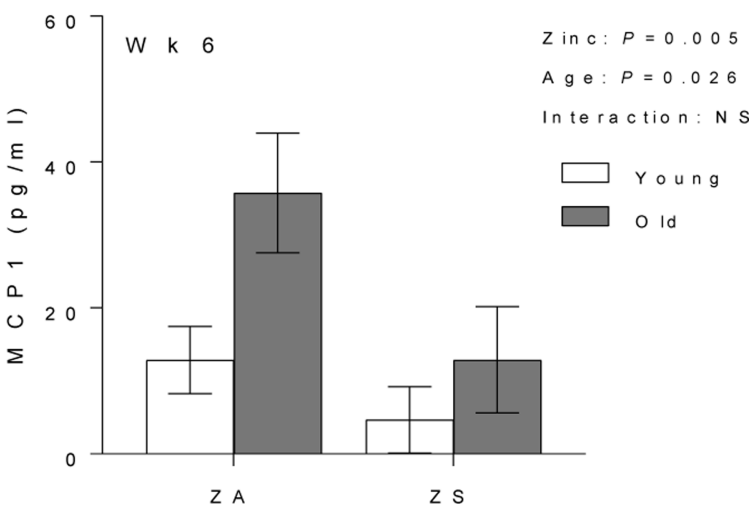

Figure 2. Dietary zinc supplementation reversed age-related decline in serum zinc and reduced whole blood MCP1 levels in old mice.

Young ( $2 \mathrm{mo}$ ) and old (24 mo) C57BL/6 mice were fed a zinc adequate (ZA) diet or zinc supplemented (ZS) diet for $6 \mathrm{wks}$. Serum zinc levels were measured by ICP-OES at the end of the study (A). Whole blood were collected at the beginning (wk 0) (B) and end of study (wk 6) (C) to determine MCP1 response in unstimulated whole blood. Data represent mean \pm SEM ( $n=7-10$ per treatment group). Two-way ANOVA was used to test for main effects of zinc status and age and their interaction. NS = not significant. 

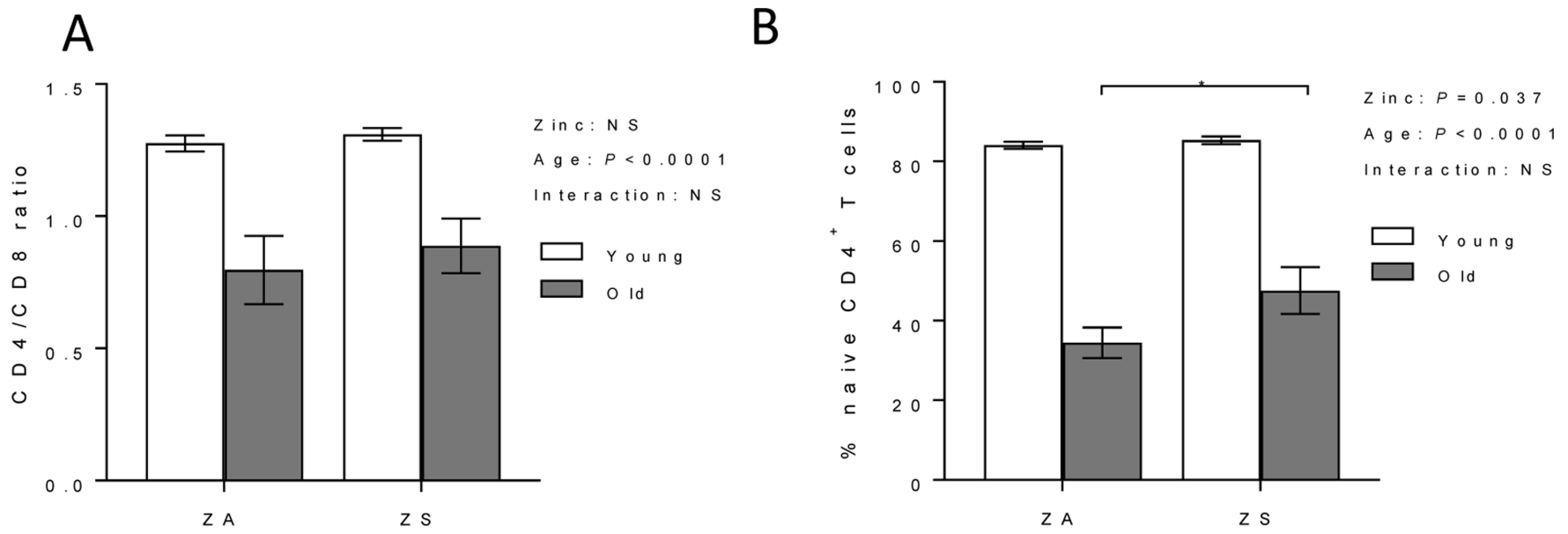

Figure 3. Zinc supplementation did not alter CD4/CD8 ratio but increased naïve $\mathrm{CD4}^{+} \mathrm{T}$ cell population in old mice.

Young (2 mo) and old (24 mo) C57BL/6 mice were fed a zinc adequate (ZA) diet or zinc supplemented (ZS) diet for 6 wks. At the end of the study, lymph node T cells were analyzed for (A) CD4/CD8 ratio, and (B) relative frequency of $\mathrm{CD}^{+}$naïve $\mathrm{T}$ cells (defined as $\mathrm{CD} 4{ }^{+} \mathrm{CD} 62 \mathrm{~L}^{\text {high }} \mathrm{CD} 44^{\text {low }} \mathrm{T}$ cells). Data represent mean $\pm \mathrm{SEM}(\mathrm{n}=7-10$ per treatment group. Two-way ANOVA was used to test for main effects of zinc status and age and their interaction, followed by Tukey's multiple comparisons test when appropriate. ${ }^{*} P<0.05$, NS $=$ not significant. 

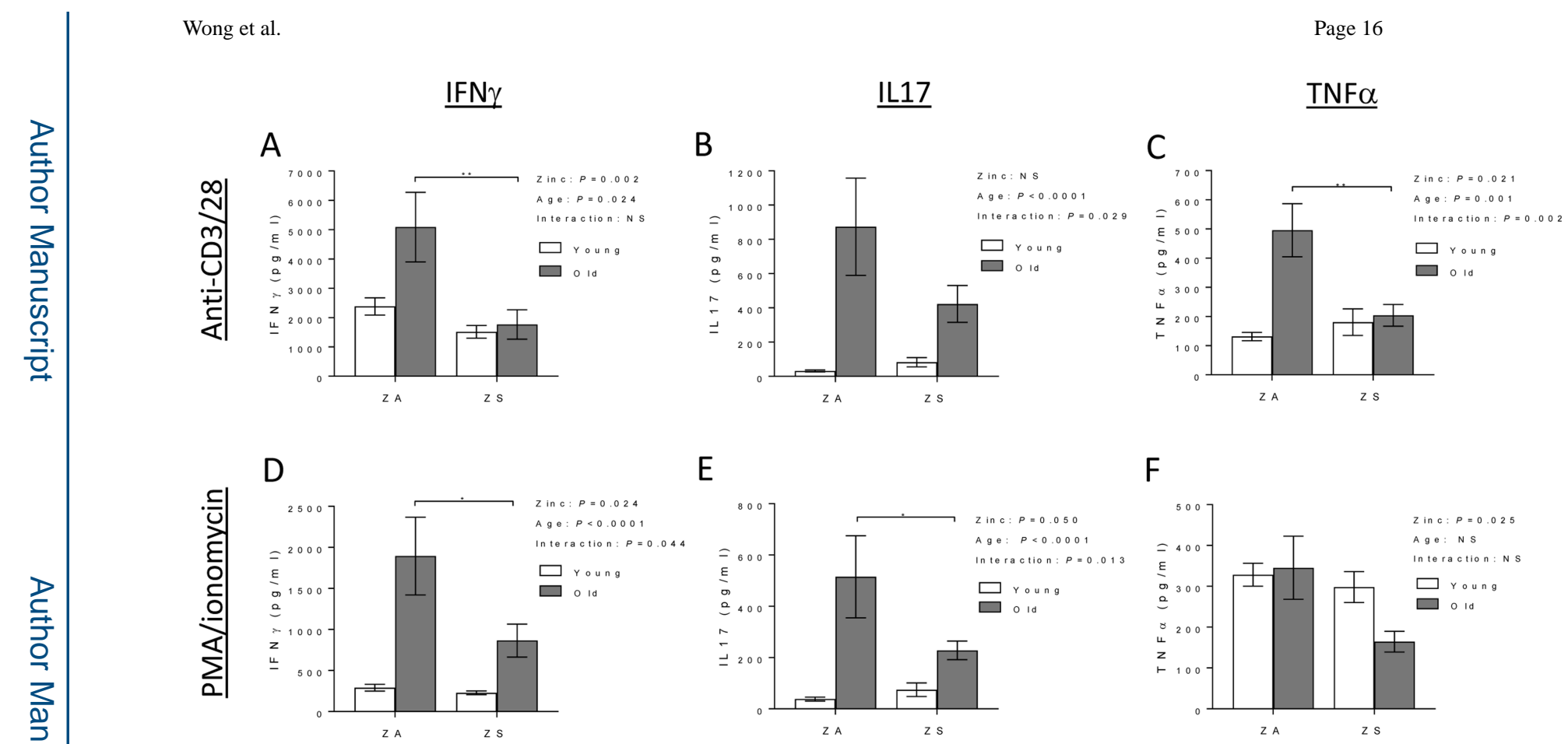

Figure 4. Age-related increase in activation-induced Th1/Th17/proinflammatory cytokines in lymph nodes were reduced with zinc supplementation in old mice.

Young (2 mo) and old (24 mo) C57BL/6 mice were fed a zinc adequate (ZA) diet or zinc supplemented (ZS) diet for 6 wks. At the end of the study, equal number of lymph node cells were stimulated ex vivo with anti-CD3 and anti-CD28 for 48h (top panels), or PMA/ ionomycin for 24h (bottom panels). Production of IFN $\gamma$ (A, D), IL17 (B, E) and TNFa (C, F) were measured. Data represent mean \pm SEM ( $n=7-10$ per treatment group). Two-way ANOVA was used to test for main effects of zinc status and age and their interaction, followed by Tukey's multiple comparisons test when appropriate. $* P<0.05, * * P<0.01, \mathrm{NS}=$ not significant. 